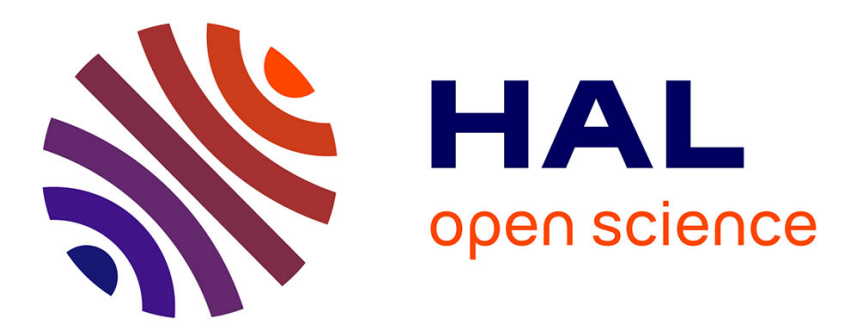

\title{
RDF/SPARQL Design Pattern for Contextual Metadata
}

Olivier Corby, Catherine Faron Zucker

\section{To cite this version:}

Olivier Corby, Catherine Faron Zucker. RDF/SPARQL Design Pattern for Contextual Metadata. IEEE/WIC/ACM International Conference on Web Intelligence, Nov 2007, Silicon Valley, United States. pp.474 - 477, 10.1109/WI.2007.162 . hal-01531172

\section{HAL Id: hal-01531172 \\ https://hal.inria.fr/hal-01531172}

Submitted on 1 Jun 2017

HAL is a multi-disciplinary open access archive for the deposit and dissemination of scientific research documents, whether they are published or not. The documents may come from teaching and research institutions in France or abroad, or from public or private research centers.
L'archive ouverte pluridisciplinaire HAL, est destinée au dépôt et à la diffusion de documents scientifiques de niveau recherche, publiés ou non, émanant des établissements d'enseignement et de recherche français ou étrangers, des laboratoires publics ou privés. 


\section{RDF/SPARQL Design Pattern for Contextual Metadata}

\author{
Olivier Corby \\ INRIA Sophia Antipolis - Méditerranée \\ FR-06902 Sophia Antipolis cedex \\ olivier.corby@sophia.inria.fr
}

\author{
Catherine Faron-Zucker \\ Université de Nice-Sophia Antipolis, I3S, \\ CNRS FR-06903 Sophia Antipolis cedex \\ faron@ polytech.unice.fr
}

\begin{abstract}
The basic principle of the Semantic Web carried by the $\mathrm{RDF}$ data model is that a collection of RDF statements coexist all together and are universally true. However some case study imply contextual relevancy and truth. The SPARQL query language is provided with patterns enabling to choose the RDF dataset against which a query is executed. This is a first step to handle contextual metadata. Based on it, we present in this paper a design pattern to handle contextual metadata hierarchically organized. This is done by means of a subst ateOf property reifying RDF entailment between contextual graphs. The substateof relation is modelled within RDF and therefore context hierarchies can be described and queried by means of SPARQL queries. We propose a slight syntactic extension to SPARQL to facilitate the query of context hierarchies, together with rewriting rules to return to standard SPARQL.
\end{abstract}

\section{Introduction}

A basic principle of the Semantic Web is to make available a collection of RDF annotations of web ressources enabling their semantic retrieval. More complex scenarios implying contextual relevance or trust require more than the RDF data model where a set of statements coexist all together and are universally true.

The SPARQL query language for RDF is provided with named graph patterns enabling to choose the RDF dataset against which a query is executed. This is a first step to handle contextual metadata. It can be used to limit the scope of an RDF statement to the context in which it is relevant to query it. Furthermore, by naming contextualized RDF graphs, they can be themselves associated with RDF metadata, enabling querying on several levels of annotations [7].

In this paper we present an RDF/SPARQL design pattern to represent and query contextual metadata. We propose to organize RDF graphs into a hierarchical structure enabling to represent inclusion of contexts. This is done by means of a subStateOf property reifying RDF entailment between contextual graphs. The advantage of defining a subStateOf property within the RDF model is that context hierarchies can be described and queried with SPARQL. We propose an extension of SPARQL to lighten the syntax of queries exploiting hierarchies of RDF datasets.

The RDF/SPARQL design pattern and SPARQL extension we present in this paper are implemented in the Corese ${ }^{1}$ RDF semantic search engine [2], [3]. CORESE has already proved itself in more than twenty semantic web applications; it is currently involved in several ongoing IST european and french projects, among which the e-WOK_HUB ANR french research project ${ }^{2}$ for which the features relative to contextual metadata will be put to work.

In the next section we present SPARQL patterns on named graphs and their use to define the notion of contextual metadata and meta-metadata. We describe in section 3 the design pattern we propose to handle contextual metadata hierarchically organized. Section 4 is dedicated to the extension of SPARQL we propose to simply handle such metadata hierarchies and section 5 to the rewriting rules enabling to return to standard SPARQL. Finally we present some case studies of context hierarchies.

\section{SPARQL Patterns on Named Graphs}

The SPARQL query language is a W3C candidate recommendation for asking and answering queries against RDF data. It offers capabilities for querying by graph patterns and retrieval of solutions is based on graph matching.

A SPARQL query is executed against an RDF dataset which represents a collection of graphs: a default graph which does not have a name, and named graphs identified by URIs. Patterns on named graphs enable to query a set of RDF graphs named by their URIs.

For instance, the following SPARQL query asks both for a pattern in an RDF graph of the dataset whose URI is bound

\footnotetext{
${ }^{1}$ http://www.inria.fr/acacia/soft/corese

${ }^{2}$ http://www.inria.fr/acacia/project/ewok
} 
to variable ? src and for a triple pattern in the default graph of the dataset stating that there is an $\mathrm{c}$ : author property attached to this graph URI bound to ? src.

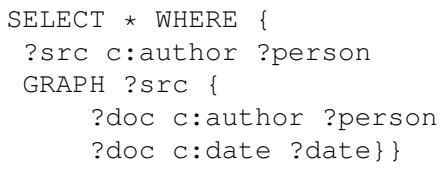

The GRAPH keyword is used to match patterns against named graphs in the query's RDF dataset; it can provide a URI to select one graph or use a variable which will range over the URIs of all named graphs in the dataset, as it is the case in our example. A SPARQL query may change the default RDF dataset to be used for matching. A new RDF dataset can be specified by using FROM NAMED clauses: it is the set of graphs referred to in the FROM NAMED clauses and the default graph is the RDF merge of these graphs.

For instance, the following SPARQL query requires to match a graph pattern against two graphs, named URI1 and URI2. In case a solution is retrieved, the name of the matching RDF graph will be provided through the binding of variable ? src1 and ? src2 used by the GRAPH keyword.

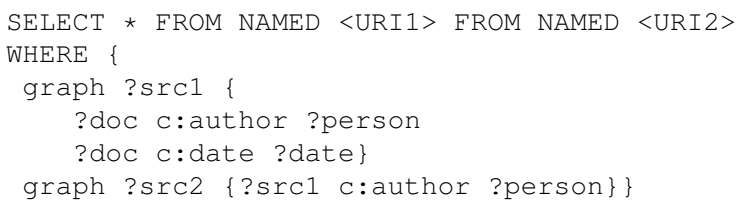

Let us note in these two examples that a SPARQL query can match different parts of its pattern against different graphs of its RDF dataset. Let us then pay special attention to the two parts of the query's pattern in both examples: one of them involves the variable denoting the graph upon which the other is to be matched. The key point here is that graph URIs are resources that can therefore be part of the RDF dataset. Hence it is possible to write and query both metadata and meta-metadata, i.e. metadata about RDF graphs named by URIs. This is the basis to explicitly tie contextual information to RDF annotations in separate RDF (meta-) annotations.

As a first conclusion, named graphs can be used to represent different contexts within which alternative metadata can be described, and these contexts themselves (their URIs) can also be represented into separate named graphs.

\section{Context Design Pattern}

SPARQL semantics considers an unstructured collection of RDF graphs for datasets. However, in some applications, we would like to model the case where some metadata are considered as universally true and some other are concurrent hypothesis or interpretations that rely on the common

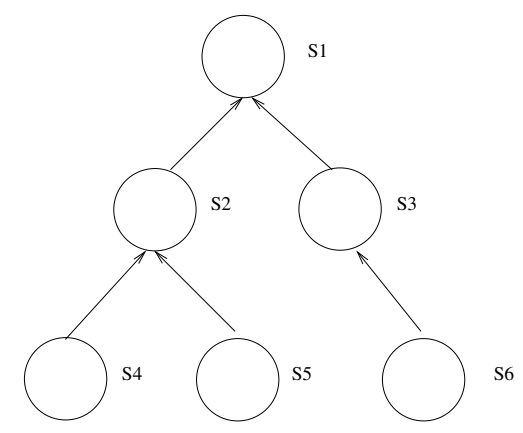

Figure 1. Example of a tree of contexts.

metadata. This would be interesting when annotating experiments (e.g. in biology) or analysis (e.g. in geology).

Hence we propose to hierarchically organize RDF datasets, based on RDFS entailment. When considering RDF datasets as contexts, the root of the hierarchy contains the common metadata that is true in any context, any other node of the hierarchy entails it. The other nodes of the hierarchy represent specific contexts; each one recursively inherits the triples of its ancestors and specializes the metadata embedded in the root context with concurrent sets of triples. To avoid the duplication of triples in this hierarchy, each node contains its own triples and inheritance is dynamically computed. This is close to the template of genus/differentia in Sowa's theory of conceptual graphs [6] for concept defintion: a concept has the graph defining its ancestor as genus and the graph specializing it, and therefore differenciating it from its ancestor, as differentia.

In the Design Pattern we propose for handling contextual metadata, to reason with a context hierarchy, we reify RDFS entailment by a transitive and reflexive relation that is represented in RDF/OWL Lite by a subStateof property between graph URIs. As a result, let us consider a tree structure of contextual metadata as depicted in figure 1. The RDF annotation below represents a snapshot of the hierarchical organization by means of occurrences of the substateof properties between graph URIs.

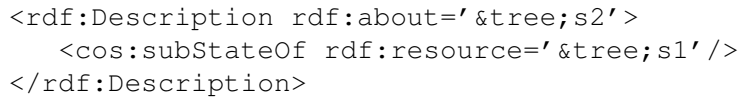

As stated before, each node contains its own triples and inheritance is dynamically computed. For instance, the context identified by $s 2$ comprises both the triples in $s 2$ and in s1 since a subStateof property holds between them.

We then base upon the SPARQL pattern on named graphs to query such a context hierarchy. Suppose that we want to match a query pattern against the $s 2$ context. We use graph variables ?s1 and ?s2 that go through all the contexts that $s 2$ is a substate of (by transitivity), including $s 2$ itself (by reflexivity) - in our example $s 2$ and $s 1$. In the 


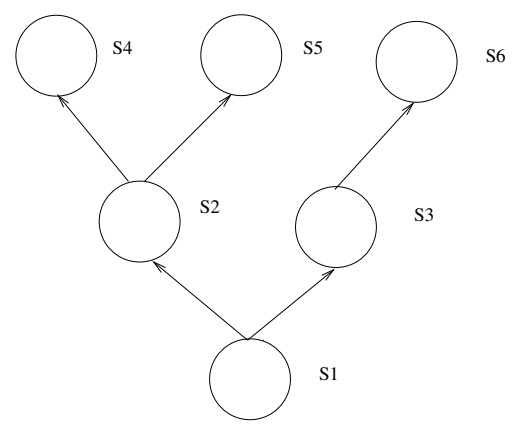

Figure 2. Example of context partonomy.

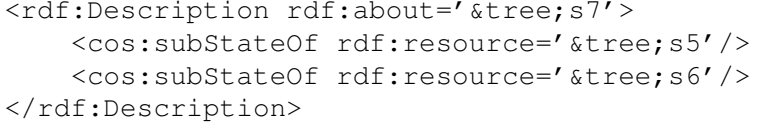

Context Partonomy In the previous part we have shown hierarchies of contexts where a node represents the common knowledge that is recursively inherited by its descendants. It is also possible to consider hierarchies where a node represents the union of its sons. In that case, the root context represents the union of all the triples of the hierarchy. Our state pattern enables to implement this use case as shown in figure 2. The state pattern below goes through all states because tree:s 1 inherits the whole hierarchy that can be considered as a partonomy:

SELECT * WHERE

STATE tree:s1 \{ PATTERN \}

However, it is not intuitive from a user point of view to describe such a partonomy upside down. Hence, we introduced a second property named subPartof defined as the inverse property of substateof.

\section{Related Work}

[1] address the problem of provenance and trust on the web and propose an extension of RDF to handle RDF graphs named by URIs, enabling RDF statements describing RDF graphs. The authors apply their work to Semantic Web publishing where named graphs allow publishers to communicate assertional intent, and to sign their graphs. Graphs are then trusted depending on their content, information about the graph, and the task the user is performing.

[7] use the notion of context to separate statements that refer to different contextual information. They describe a practical solution to explicitly tie contextual information to RDF statements. They identify SPARQL as the query language satisfying their requirements with its patterns on named graphs, however they do not propose any extension of RDF or SPARQL.

\section{Conclusion and on going Work}

In this paper we have presented an RDF/SPARQL design pattern to represent contextual metadata using a hierarchy of contexts represented as named graphs and ordered by RDFS entailment. We have proposed an operational mechanism for expressing and handling contextual metadata through the extension of SPARQL with a STATE pattern and its compilation into standard SPARQL.

This SPARQL extension has been implemented in the Corese semantic search engine and will be used in the eWOK_HUB ANR french research project dedicated to geo sciences problems where alternative interpretations of geological data must be represented and reasoned about.

We argue that contextual reasoning is a key issue for intelligent web systems, especially for social networks, intelligent human-web interaction, web trust, etc.. Many applications show us the benefits that would provide the extension of RDF and SPARQL to define, exchange and query identified sources of RDF statements.

Acknowledgement We would like to cite the former Smeci project from INRIA which introduced state trees for rule based reasoning and acknowledge Pierre Haren for these fructuous ideas as well as Rose Dieng for metareasoning on state trees.

\section{References}

[1] J. Carroll, C. Bizer, P. Hayes and P. Stickler. Named Graphs, Provenance and Trust. 14th World Wide Web Conference, Chiba, Japan, pages 613-622, 2005.

[2] O. Corby, R. Dieng, C. Faron-Zucker, F. Gandon. Searching the Semantic Web: Approximate Query Processing based on Ontologies, IEEE Intelligent Systems 21(1), 2006.

[3] O. Corby, C. Faron-Zucker. Implementation of SPARQL based on Graph Homomorphism. 15th International Conference on Conceptual Structures, Sheffield, UK, 2007.

[4] G. Kahn. Natural Semantics. RR-0601 INRIA, 1987.

[5] E. Prud'hommeaux, A. Seaborne, SPARQL Query Language for RDF, W3C Candidate Recommendation, 2007. http://www.w3.org/TR/rdf-sparql-query

[6] J.F. Sowa, Conceptual Structures: Information Processing in Mind and Machine, Addison-Wesley, 1984.

[7] H. Stoermer, I. Palmisano, D. Redavid, L. Iannone, P. Bouquet and G. Semeraro. RDF and Contexts: Use of SPARQL and Named Graphs to Achieve Contextualization. 1st Jena User Conference, Bristol, UK, 2006. 